Supporting Information for

\title{
A Guided Ion Beam and Quantum Chemical Investigation of the Thermochemistry of Thorium Dioxide Cations: \\ Thermodynamic Evidence for Participation of f Orbitals in Bonding
}

\author{
P. B. Armentrout ${ }^{1 *}$ Kirk A. Peterson ${ }^{2}$ \\ ${ }^{1}$ Department of Chemistry, University of Utah, Salt Lake City, UT 84112-0850 USA \\ ${ }^{2}$ Department of Chemistry, Washington State University, Pullman, Washington 99164- \\ 4630, USA
}

\section{Thermochemistry for $\mathrm{TiO}_{2}{ }^{+}$}

Information on the titanium monoxide and dioxide cations is available from several sources. Hildenbrand determined the $0 \mathrm{~K}$ bond dissociation energy of TiO as $662.7 \pm 6.3$ $\mathrm{kJ} / \mathrm{mol}=6.87 \pm 0.07 \mathrm{eV}^{1}$ and Pedley and Marshall report a similar TiO bond energy of 668 $\pm 9 \mathrm{~kJ} / \mathrm{mol}=6.92 \pm 0.09 \mathrm{eV} .{ }^{2}$ The best value for IE(TiO) comes from Sappey et al., $6.819 \pm$ $0.006 \mathrm{eV},{ }^{3}$ and that for IE(Ti $)=6.828120 \pm 0.000012 \mathrm{eV}^{4}{ }^{4}$ Combining these IEs with $\mathrm{D}_{0}(\mathrm{TiO})$ from Hildenbrand or Pedley and Marshall yields $\mathrm{D}_{0}\left(\mathrm{Ti}^{+}-0\right)=6.88 \pm 0.07 \mathrm{eV}$ or $6.93 \pm 0.09$ $\mathrm{eV}$, which are in excellent agreement with the value obtained directly from guided ion beam tandem mass spectrometry studies of the reaction of $\mathrm{Ti}^{+}$with $\mathrm{CO}, 6.88 \pm 0.07 \mathrm{eV} .^{5}$

For the dioxide, the atomization energy of $\mathrm{TiO}_{2}$ was reported by Hildenbrand as $\mathrm{AE}_{0}$ $=1271.9 \pm 11.7 \mathrm{~kJ} / \mathrm{mol}=13.18 \pm 0.12 \mathrm{eV},{ }^{1}$ and Balducci et al. report $\mathrm{AE}_{0}\left(\mathrm{TiO}_{2}\right)=1260 \pm$ $12 \mathrm{~kJ} / \mathrm{mol}=13.06 \pm 0.12 \mathrm{eV}^{6}{ }^{6}$ Using electron ionization threshold measurements, Hildenbrand determined ionization energies of $\operatorname{IE}(\mathrm{TiO})=6.70 \pm 0.10 \mathrm{eV}$ and $\mathrm{IE}\left(\mathrm{TiO}_{2}\right)=9.54$ $\pm 0.10 \mathrm{eV} .{ }^{1}$ Balducci et al. report a similar but much less precise $\operatorname{IE}\left(\mathrm{TiO}_{2}\right)=9.5 \pm 0.5 \mathrm{eV},{ }^{6}$ whereas Rauh and Ackermann report $10.2 \pm 0.2 \mathrm{eV} .^{7}$ To obtain the atomization energy of $\mathrm{TiO}_{2}{ }^{+}$, we combine $\mathrm{IE}\left(\mathrm{TiO}_{2}\right)=9.54 \pm 0.10 \mathrm{eV}$ with $\mathrm{IE}(\mathrm{Ti})$ and $\mathrm{D}_{0}\left(\mathrm{TiO}_{2}\right)$ from either 
Hildenbrand and Murad or Balducci et al. to obtain $\mathrm{AE}_{0}=10.47 \pm 0.16 \mathrm{eV}$ or $10.35 \pm 0.16$ $\mathrm{eV}$. Using $\mathrm{D}_{0}\left(\mathrm{Ti}^{+}-0\right)=6.88 \pm 0.07 \mathrm{eV}$, this leads to $\mathrm{D}_{0}\left(\mathrm{OTi}^{+}-0\right)=3.59 \pm 0.18 \mathrm{eV}$ or $=3.47 \pm$ $0.18 \mathrm{eV}$. We adopt the weighted mean of these two values, $3.53 \pm 0.13 \mathrm{eV}$.

\section{References}

1. Hildenbrand, D. L. Mass spectrometric studies of the thermochemistry of gaseous TiO and $\mathrm{TiO}_{2}$. Chem. Phys. Lett. 1976, 44, 281-284.

2. Pedley, J. B.; Marshall, E. M. Thermochemical Data for Gaseous Monoxides. J. Phys. Chem. Ref. Data 1983, 12, 967-1031.

3. Sappey, A. D.; Eiden, G.; Harrington, J. E.; Weisshaar, J. C. Vibronic structure of $\mathrm{TiO}^{+}$ from multiphoton ionization photoelectron spectroscopy. J. Chem. Phys. 1989, 90, 14151428.

4. $\quad$ Kramida, A.; Ralchenko, Y.; Reader, J.; Team, N. A., NIST Atomic Spectra Database (ver. 5.7.1), [Online]. Available: http://physics.nist.gov/asd. National Institute of Standards and Technology, Gaithersburg, MD.: 2012.

5. $\quad$ Clemmer, D. E.; Elkind, J. L.; Aristov, N.; Armentrout, P. B. Reaction of $\mathrm{Sc}^{+}, \mathrm{Ti}^{+}$, and $\mathrm{V}^{+}$ with CO. $\mathrm{MC}^{+}$and $\mathrm{MO}^{+}$Bond Energies. J. Chem. Phys. 1991, 95, 3387-3393.

6. Balducci, G.; Gigli, G.; Guido, M. Mass spectrometric study of the thermochemistry of gaseous $\mathrm{EuTiO}_{3}$ and $\mathrm{TiO}_{2}$. J. Chem. Phys. 1985, 83, 1909-1912.

7. Rauh, E. G.; Ackermann, R. J. First Ionization Potentials of Some Refractory Oxide Vapors J. Chem. Phys. 1974, 60, 1396-1400. 
Table S1. Molecular parameters, bond energies, and relative energies of $\mathrm{ThO}^{+}$and $\mathrm{ThO}_{2}{ }^{+}$calculated at various levels of theory. ${ }^{\mathrm{a}}$

\begin{tabular}{|c|c|c|c|c|c|c|c|c|c|}
\hline species & State & $\mathrm{s}(\mathrm{s}+1)$ & Basis set & $\mathrm{r}\left(\mathrm{Th}^{+}-\mathrm{O}\right)(\AA)$ & $\angle \mathrm{OThO}\left(^{\circ}\right)$ & $v\left(\mathrm{~cm}^{-1}\right)$ & $D_{0} / \mathrm{E}_{\mathrm{rel}}($ & & \\
\hline & & & & & & & B3LYP & $\mathrm{MP} 2^{\mathrm{b}}$ & $\operatorname{CCSD}(T)^{\mathrm{b}}$ \\
\hline \multirow[t]{4}{*}{$\mathrm{ThO}^{+}$} & ${ }^{2} \Sigma^{+}$ & 0.75 & Seg. SDD & 1.800 & & 962 & 9.160 & & \\
\hline & & 0.75 & cc-pwCVTZ & 1.809 & & 961 & 9.305 & 9.912 & 9.113 \\
\hline & & 0.75 & cc-pwCVQZ & 1.808 & & 961 & 9.307 & 10.064 & 9.262 \\
\hline & & & $\mathrm{CBS}^{\mathrm{c}}$ & & & & & 10.150 & 9.347 \\
\hline \multirow[t]{12}{*}{$\mathrm{ThO}_{2}^{+}$} & ${ }^{2} \Sigma^{+}$ & 0.75 & Seg. SDD & $1.867(2)$ & 180.0 & $37(2), 633,753$ & 4.831 & & \\
\hline & & 0.80 & cc-pwCVTZ & $1.877(2)$ & 180.0 & $34(2), 627,757$ & 4.889 & 4.849 & 4.572 \\
\hline & & 0.80 & cc-pwCVQZ & $1.876(2)$ & 180.0 & $33(2), 625,756$ & 4.883 & 4.968 & 4.698 \\
\hline & & & $\mathrm{CBS}^{\mathrm{c}}$ & & & & & 5.037 & 4.771 \\
\hline & ${ }^{2} \mathrm{~A}_{1}$ & 0.75 & Seg. SDD & $2.029(2)$ & 43.2 & $569,616,882$ & 2.795 & & \\
\hline & & 0.75 & cc-pwCVTZ & $2.033(2)$ & 42.8 & $553,615,881$ & 3.210 & 3.383 & 3.125 \\
\hline & & 0.75 & cc-pwCVQZ & $2.033(2)$ & 43.1 & $575,619,881$ & 2.767 & 2.728 & 2.589 \\
\hline & & & $\mathrm{CBS}^{\mathrm{c}}$ & & & & & 2.508 & 2.439 \\
\hline & ${ }^{4} \mathrm{~B}_{2}$ & 3.77 & Seg. SDD & $2.094(2)$ & 112.8 & $86,502,585$ & 4.401 & & \\
\hline & & 3.77 & cc-pwCVTZ & $2.094(2)$ & 112.8 & $86,502,585$ & 4.313 & 5.202 & 4.380 \\
\hline & & 3.77 & cc-pwCVQZ & $2.094(2)$ & 112.8 & $86,502,585$ & 4.304 & 5.289 & 4.477 \\
\hline & & & $\mathrm{CBS}^{\mathrm{c}}$ & & & & & 5.343 & 4.537 \\
\hline
\end{tabular}

a Structures optimized at B3LYP/basis set level of theory. ${ }^{\mathrm{b}}$ Single point energies calculated using B3LYP optimized structures and the same basis set. ${ }^{\mathrm{c}}$ Complete basis set extrapolation. 
Table S2. Absolute energies $\left(E_{h}\right)$ for calculations.

\begin{tabular}{|c|c|c|c|c|c|}
\hline Species & State & Basis set & B3LYP & MP2 & $\operatorname{CCSD}(\mathrm{T})$ \\
\hline \multirow[t]{4}{*}{$\mathrm{O}$} & ${ }^{3} \mathrm{P}$ & Seg. SDD & -75.099660 & & \\
\hline & & cc-pwCVTZ & -75.094975 & -74.964295 & -74.9836308 \\
\hline & & cc-pwCVQZ & -75.099660 & -74.979102 & -74.9972776 \\
\hline & & $\mathrm{CBS}^{\mathrm{c}}$ & & -74.986254 & -75.0037605 \\
\hline \multirow[t]{8}{*}{$\mathrm{Th}^{+}$} & ${ }^{2} \mathrm{D}$ & Seg. SDD & -407.330159 & & \\
\hline & & cc-pwCVTZ & -406.496623 & -405.911193 & -405.8646600 \\
\hline & & cc-pwCVQZ & -406.497477 & -405.969966 & -405.9207185 \\
\hline & & $\mathrm{CBS}^{\mathrm{c}}$ & & -406.003739 & -405.9529256 \\
\hline & ${ }^{4} \mathrm{~F}$ & Seg. SDD & -407.323626 & & \\
\hline & & cc-pwCVTZ & -406.489171 & -405.907067 & -405.8583770 \\
\hline & & cc-pwCVQZ & -406.489965 & -405.965867 & -405.9148443 \\
\hline & & $\mathrm{CBS}^{\mathrm{c}}$ & & -405.999681 & -405.9473122 \\
\hline \multirow[t]{4}{*}{$\mathrm{ThO}^{+}$} & ${ }^{2} \Sigma^{+}$ & Seg. SDD & -482.762114 & & \\
\hline & & cc-pwCVTZ & -481.928303 & -481.237794 & -481.1790920 \\
\hline & & cc-pwCVQZ & -481.933848 & -481.316992 & -481.2546969 \\
\hline & & $\mathrm{CBS}^{\mathrm{c}}$ & & -481.361135 & -481.2967664 \\
\hline $\mathrm{ThO}_{2}{ }^{+}$ & ${ }^{2} \Sigma^{+}$ & Seg. SDD & -558.040429 & & \\
\hline
\end{tabular}




\begin{tabular}{|c|c|c|c|c|}
\hline & cc-pwCVTZ & -557.204051 & -556.381416 & -556.3318530 \\
\hline & cc-pwCVQZ & -557.214070 & -556.479761 & -556.4257196 \\
\hline & $\mathrm{CBS}^{\mathrm{c}}$ & & -556.533599 & -556.4769736 \\
\hline${ }^{2} \mathrm{~A}_{1}$ & Seg. SDD & -557.939090 & & \\
\hline & cc-pwCVTZ & -557.087449 & -556.258440 & -556.2183730 \\
\hline & cc-pwCVQZ & -557.113823 & -556.380950 & -556.3319963 \\
\hline & $\mathrm{CBS}^{\mathrm{c}}$ & & -556.442875 & -556.388791 \\
\hline${ }^{4} B_{2}$ & Seg. SDD & -557.878299 & & \\
\hline & cc-pwCVTZ & -557.044905 & -556.18960 & -556.1702440 \\
\hline & cc-pwCVQZ & -557.055286 & -556.28478 & -556.2605718 \\
\hline & $\mathrm{CBS}^{\mathrm{c}}$ & & -556.336640 & -556.3096336 \\
\hline
\end{tabular}

